\title{
BMJ Open Gender-based violence and absent fathers: a scoping review protocol
}

\section{Yandisa Sikweyiya,,${ }^{1,2}$ Mzikazi Nduna, ${ }^{3}$ Nelisiwe Khuzwayo, ${ }^{4}$ Andile Mthombeni, ${ }^{3}$ Tivani Phosa Mashamba-Thompson ${ }^{4}$}

To cite: Sikweyiya $Y$, Nduna M, Khuzwayo N, et al. Gender-based violence and absent fathers: a scoping review protocol. BMJ Open 2016;6:e010154.

doi:10.1136/bmjopen-2015010154

- Prepublication history for this paper is available online To view these files please visit the journal online (http://dx.doi.org/10.1136/ bmjopen-2015-010154).

Received 1 October 2015 Revised 31 March 2016 Accepted 26 April 2016

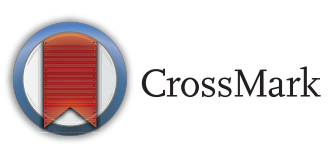

${ }^{1}$ Gender and Health Research Unit, Medical Research Council, Pretoria, South Africa

${ }^{2}$ School of Public Health, University of the Witwatersrand, Johannesburg, South Africa ${ }^{3}$ School of Psychology, University of the Witwatersrand, Johannesburg, South Africa ${ }^{4}$ Discipline of Public Health Medicine, School of Nursing and Public Health, University of KwaZulu Natal, Durban, South Africa

Correspondence to Dr Yandisa Sikweyiya; yandisa.sikweyiya@mrc.ac.za

\section{ABSTRACT}

Introduction: Gender-based violence (GBV) and absent fathers are two epidemics that affect women and children in sub-Saharan Africa. However, the understanding of the complex links between GBV and absent fathers is currently inadequate. The aim of the study is to provide an overview of documented evidence that links GBV and absent fathers as well as identifies areas that require systematic review and where more primary research is needed.

Methods and analysis: The search strategy for this scoping review study will involve electronic databases including: Academic Search Premier, Ingenta, Kluwer Online, PsycARTICLES (EBSCO), PsycINFO (EBSCO), Social Work Abstracts and Sociological Collection. The studies will be mapped in 2 stages: stage 1 will map studies descriptively by focus and method; stage 2 will involve additional inclusion criteria, quality assessment and data extraction undertaken by two reviewers in parallel. A thematic analysis of the studies will be carried out to extract relevant outcomes using NVIVO.

Discussion: We anticipate finding a large number of studies on GBV diagnostic interventions in subSaharan Africa which, once summarised, will be useful to guide future research. The protocol for the scoping review has been registered in PROSPERO.

Dissemination: The study will be disseminated electronically and in print. It will also be presented to conferences related to GBV, Father Connections and Children's Health.

\section{PROSPERO registration number:} CRD42015022094.

\section{INTRODUCTION}

Empirical evidence suggests that the prevalence of gender-based violence (GBV) is high around the world. ${ }^{12}$ Findings of the WHO's multicountry study revealed that between $13 \%$ and $61 \%$ of ever partnered women have experienced an array of lifetime physical violence. $^{2}$ The $\mathrm{WHO}^{3}$ further reports that intimate partner violence (IPV) is the most common form of GBV. This form of violence comprises physical, sexual and emotional abuse and controlling behaviour by a current or former intimate partner or spouse. ${ }^{4}$

Studies show that GBV disproportionally affects women and children, and that it is mainly perpetrated by men. ${ }^{15}$ Authors argue that GBV mostly arises from the unequal power relationships between men and women, and has a devastating impact on the lives of the victims, mainly women and children. ${ }^{6}$ GBV negatively impacts the physical, mental and sexual health of the victims, as well as the social and economic aspects of their lives. ${ }^{2} 7$ For example, there is emerging evidence that GBV may result in children growing up without their biological fathers. ${ }^{8}$

There is a paucity of literature on father absence in Africa. ${ }^{9}$ Published data suggest that between $30 \%$ and $50 \%$ of South African children grow up without their biological fathers, with black African children being the most affected. ${ }^{10}$ Father absence is a term that is rarely defined in the literature. ${ }^{9}$ However, studies have shown that 'fathers can be absent in the lives of their children fully, partially, economically and emotionally'. ${ }^{11}$ The term 'father absence' in this study refers to biological fathers who are absent in the lives of their children due to disappearance in the early stages of the child's life or those who are known to be alive or perceived to be alive but, since the early stages of the child's life, have been emotionally and economically absent leading to children feeling neglected or abandoned. ${ }^{11}$ Studies report varying reasons for father absence, and Nduna and Jewkes $^{12}$ suggest that the rape of a woman by a stranger may lead to father absence in direct ways, as a father may be genuinely unknown to the mother. Other studies show that in cases of denied paternity or unacknowledged pregnancies, the mother may have no confidence in introducing the alleged father to his child, leading to father absence. ${ }^{13-15}$ Furthermore, qualitative studies conducted in South Africa show that domestic violence may lead to a marriage or relationship break-up leading to father absence. ${ }^{16}{ }^{17}$ It is well documented in the literature that children who grow up with an absent biological father tend to display more behavioural problems and often experience 
more life trauma and distress compared to children who grow up residing with both parents. ${ }^{9}$ However, little is known about whether and how father absence predisposes children who grow up with absent biological fathers to GBV. Notwithstanding this, evidence from a few studies conducted in South Africa suggests that young people who grew up with absent fathers tend to be treated differently (compared to other children who have involved fathers) in their maternal homes, or often suffer physical, sexual and emotional abuse perpetrated by significant people in their lives (eg, maternal uncles, aunts and/or maternal grandparents). However, it is currently not known how prevalent this is in South Africa.

While there is scant literature on this topic in South Africa, mounting evidence suggests that growing up with an absent father is a risk factor for GBV perpetration by boys/men. ${ }^{18}$ Studies have demonstrated that men who perpetrate violence against women are likely to be those who did not co-reside with their biological fathers, had poor father-son relations or did not even know their fathers. ${ }^{19}$ Other studies have shown that girls with absent fathers are likely to have poor heterosexual adjustment, dissatisfying and violent sexual relationships. ${ }^{20}$

The literature and evidence above suggest that GBV and absent fathers might be two epidemics that excessively affect women and children in South Africa. However, the understanding of the complex links between GBV and absent fathers is currently inadequate. This underscores the need for research to explore the links between these two phenomena and build knowledge around this area. The main aim of the study is to provide an overview of documented evidence that suggests a link between GBV and absent fathers in sub-Saharan Africa, as well as identifies areas that require systematic review and where more primary research is needed. In order to fulfil the above aim, the following objectives will guide the study:

- To review evidence that the absent father phenomenon leads to perpetration of GBV by boys and men.

- To examine evidence that the absent father phenomenon contributes to GBV victimisation of girls.

- To examine evidence that the absent father phenomenon contributes to GBV victimisation of boys.

- To determine evidence that GBV leads to absent fathers in children's lives.

The main research question: is there a link between GBV and absent fathers?

Research subquestions are as follows:

1. Is there evidence that the absent father phenomenon leads to perpetration of GBV by boys and men?

2. Is there evidence that the absent father phenomenon contributes to GBV victimisation of girls?

3. Is there evidence that the absent father phenomenon contributes to GBV victimisation of boys?

4. Is there evidence that GBV leads to absent fathers in children's lives?
Eligibility of research question

Materials and methods

This scoping review protocol has been registered and published in the PROSPERO international prospective register of systematic reviews. It is registered under the following number: CRD42015022094

\section{Study selection}

Peer-reviewed studies and grey literature that addresses the research question will be included in the search. An electronic search of the database of all studies with various study designs will be included, as well as the grey literature. Furthermore, when necessary, expert opinions will be sought. The databases that will be used to search for literature include: Academic Search Premier, Ingenta, Kluwer Online, PsycARTICLES (EBSCO), PsycINFO (EBSCO), Social Work Abstracts and Sociological Collection.

\section{Data extraction}

The research team will carry out a narrative review or analytical method to extract background or process oriented information from each included study through the following process (see figure 1):

- Collectively developing the data-charting form and determining the variables and themes to extract in order to answer the research question.

- Updating charting data.

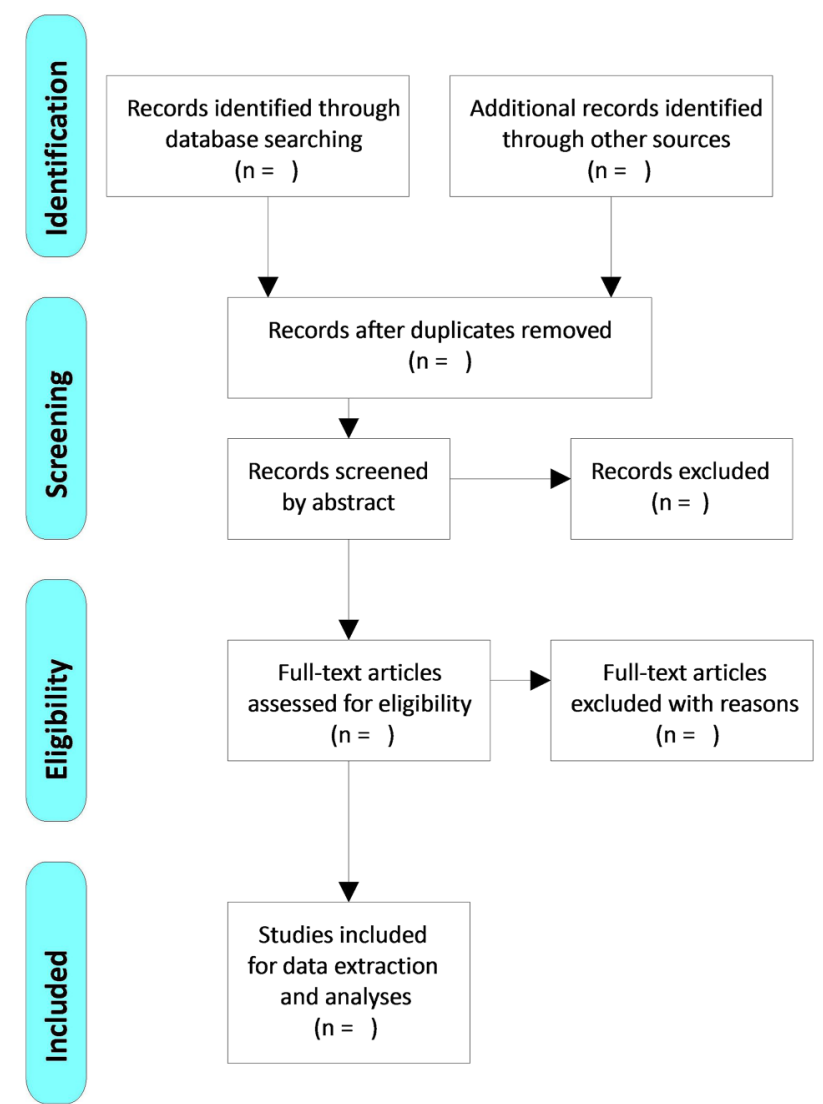

Figure 1 A flow chart showing phases of the literature search for extraction of the most specific literature for the review. 
Two independent reviewers will extract data from all eligible studies in duplicate using a standardised and piloted data abstraction spreadsheet. A third reviewer will be consulted if necessary to reach consensus. Bibliographic details, study design, number of participants, intervention (s), comparison(s), study setting, funding source and conclusions for the primary and secondary outcomes of interest will be extracted. Information specific to the father's absence, description of the intervention (GBV) and geographic location of the study will also be extracted. Quality appraisal will not be performed on studies included in this scoping review study.

- A thematic analysis will be carried out collectively by researchers to extract relevant outcomes using NVIVO

Inclusion and exclusion criteria (see table 1) were developed to ensure a sufficient level of comparability across GBV in families and communities raising children in the absence of fathers and those raising children in the presence of fathers.

Inclusion criteria

- Evidence published in the English language.

- Evidence from published relevant interventions and studies in sub-Saharan Africa.

- Studies with focus on families with children with absent fathers.

- Research reporting on intervention with children 18 years of age and younger.

- Literature published from January 1990 up to June 2015

- Review articles including: peer-reviewed journal articles, systematic reviews, scoping reviews, meta-analysis and rapid reviews.

- Grey literature including: government and nongovernmental organisation reports and academic dissertations.
- Full reports of studies including: qualitative, quantitative and mixed methods studies will be considered.

- All study designs will be considered including crosssectional qualitative and quantitative studies, randomised controlled trials, and quasi-experimental designs and pilot studies.

Exclusion criteria

- Evidence from countries outside sub-Saharan Africa will be excluded because the cultures and nature of GBV may be substantially different from our target group.

- Evidence from conflict or post-conflict settings (see definitions below) will be excluded as causes or circumstances under which fathers become absent during and/or immediately after conflict may be different in nature when equated to those dynamics in non-conflict settings.

- Evidence where the impact of fathers' absence could not be differentiated from the impact of a broader interference/intervention.

- Non-English publications will be excluded.

- Studies published before January 1990 and after June 2015.

- Studies with no focus on children.

- Studies reporting father absence due to incarceration.

- Studies reporting father absence due to death.

- Studies reporting father absence due to travel for long duration for work but involved in child's life.

- Studies on orphaned and institutionalised children.

During the keyword search (see table 2), keywords will be combined into a phrase including Boolean (AND, OR) terms, a sample as demonstrated below (as this will be an iterative process and will be documented in the analysis and write-up):

\begin{tabular}{|c|c|}
\hline Criteria & Determinants \\
\hline Population & $\begin{array}{l}\text { The population for this study will be children raised without, and with no connection to, their biological fathers. } \\
\text { Children who are orphans or institutionalised will be excluded from this study. An orphaned child is defined as } \\
\text { a young person below the age of } 18 \text { years whose biological parents have died. The death of both biological } \\
\text { parents could confound the links between GBV and absent fathers. } \\
\text { An institutionalised child is defined as a young person below the age of } 18 \text { years who has been placed in an } \\
\text { institution (eg, children's shelter or orphanage). The intervention (ie, professional support, place of safety or } \\
\text { protection, or provision of other critical services) of the institution on children placed in such institutions could } \\
\text { muddle the links between GBV and the absent father. }\end{array}$ \\
\hline Intervention & Absence of fathers, gender-based violence \\
\hline Comparisons & Children raised with connection to their fathers \\
\hline Outcomes & Gender-based violence, father absence \\
\hline Study setting & $\begin{array}{l}\text { Sub-Saharan Africa } \\
\text { While we have largely cited literature from South Africa in this protocol, owing to the paucity of literature on } \\
\text { father absence in Africa, we propose to extend the scope of this review to include studies from other } \\
\text { sub-Saharan countries. The rationale for extending the review to sub-Saharan Africa is to increase the pool of } \\
\text { studies to include in this scoping review study. Furthermore, we believe that countries in the sub-Saharan } \\
\text { region are not too dissimilar in terms of socioeconomic status and social dynamics that lead to father absence. }\end{array}$ \\
\hline
\end{tabular}


Table 2 Electronic search record

\begin{tabular}{llll}
\hline Keyword & $\begin{array}{l}\text { Search } \\
\text { engine } \\
\text { used }\end{array}$ & $\begin{array}{l}\text { Number of } \\
\text { publications } \\
\text { retrieved }\end{array}$ \\
\hline
\end{tabular}

('Gender-based violence' OR 'Domestic Violence' OR 'Intimate partner violence' OR 'Partner Violence' OR 'Spousal violence' OR 'Dating violence' OR 'Child sexual abuse' OR 'Violence against women' AND 'Father absence' OR 'Absent and unknown fathers' OR 'Absent but known fathers' OR 'Absent and undisclosed fathers' OR 'Unknown and deceased fathers' OR 'Known but physically absent fathers' OR 'Uninvolved fathers' OR 'Father not co-residing')

All researchers will keep an updated record of the number of publications identified and date during each session of the literature search using table 2 below.

\section{Collating, summarising and reporting results}

The principal aim of this study is to scope the existing evidence and summarise the findings as presented across articles. The research team will meet to carry out a thematic analysis of the studies and provide an overview of all the data reviewed. This process will include the following steps: First, the bibliographic details, study design, number of participants, intervention(s), comparison(s), study setting and funding source of the included studies will be examined. Second, results of the studies on perpetration of GBV by boys and men, GBV victimisation of girls, GBV victimisation of boys, on evidence whether GBV leads to absent fathers, and on whether father absence leads to GBV will be coded by all authors independently. When all the included studies have been explored and coded, authors will come together and interrogate the resulting themes and critically examine their relationship to the research question. Further to this, the study team will scrutinise the meanings of the findings as they relate to the overall aim of the study, and discuss the implications for future research, practice and policy.

\section{DISCUSSION}

The proposed scoping review will generate findings that will aid in describing the links between GBV and absent fathers in sub-Saharan Africa. While this area has received little research attention, emerging evidence from South Africa suggests that children who grow up with absent fathers may be at an increased risk of experiencing various forms of GBV in their lives. However, the understanding of the links between GBV and absent fathers is currently inadequate. For example, there is little understanding on how prevalent the abuse of children with absent fathers is, what the factors are that lead to abuse, and what the nature is of the relationship between GBV and absent fathers. This review will enable the authors to answer these questions and further elucidate what is known and unknown about the links between these phenomena in the sub-Saharan region.

Specific targeted research on absent fathers and its effect on the psychological and social health of children in sub-Saharan Africa is still in its infancy. Until now, we are not aware of any review or empirical studies that have been conducted in sub-Saharan Africa to specifically look at the question addressed in the current proposal. As such, the findings of this review will contribute to knowledge on this topic area and impact practice, policy and research in the area of father connections, fatherhood and child health.

Owing to contextual dynamics, we resolved to exclude literature and studies from other regions of Africa. We perceive that the causes and circumstances that lead to father absence in conflict or post-conflict settings, for example, may be different in nature when compared to those factors in politically stable countries. As such, we argue that if we include literature and studies from politically unstable countries, we risk distorting the findings on the links between GBV and absent fathers in this review. Also, emotional absence while co-residing with the father is not considered as this is a different form of absence. The study will also exclude incarcerated fathers and institutionalised children as the disconnection to fathers may result in factors other than GBV. A similar reasoning was applied to the exclusion of orphaned children.

Results from this scoping review will be useful to policymakers, academics, researchers and programme implementers who work with families and children in sub-Saharan Africa, and other similar settings. Evidence generated in this scoping review will be a critical base on which primary prevention strategies may be built. Furthermore, this work will be critical in identifying research gaps in the father connections literature, as well as highlight areas where primary empirical work and systematic reviews are required. Academics in higher institutions of learning in South Africa have increasingly pointed to the need for methodical approaches of reviewing literature by postgraduate students, as such approaches offer a systematic way of reviewing and synthesising the literature and enhance the quality of the outputs. Thus, the methodology described in this scoping review protocol will be essential for teaching purposes at higher institutions of learning in South Africa and elsewhere. Findings of this review will become pertinent to researchers as evidence for the need for more work in this area and used to justify the need to fund this work.

\section{Strengths and limitations of this study}

The study will only include literature published in English. Therefore, studies published in other languages will be missed. To the best of our knowledge, the research area of father absence is relatively new in Africa; as such, there may be little published work that 
directly or indirectly looks at the links between GBV and father absence. While this may be a possible limitation in terms of the amount of data for this scoping review study, it may be an important finding of this study and a basis for calling for more research on this area.

\section{Definition of key terms}

GBV: Gender-based violence will include intimate partner violence, non-intimate partner violence, domestic violence, BUT will exclude gender-based violence that occurs in crisis situations (eg, conflict or postconflict settings).

GBV: Means sexual, emotional, physical and economical violence, BUT excludes sexual harassment

VAW: Violence against women will include "any act of gender-based violence that results in, or is likely to result in, physical, sexual or psychological harm or suffering to women, including threats of such acts, coercion or arbitrary deprivation of liberty, whether occurring in public or in private life." ${ }^{21}$

IPV: Intimate partner violence will include "any range of sexually, psychologically, and physically coercive acts used against adult and adolescent women by current or former male intimate partners". ${ }^{22}$

Child abuse: will include "all forms of physical and/or emotional ill-treatment, sexual abuse, neglect or negligent treatment or commercial or other exploitation, resulting in actual or potential harm to the child's health, survival, development or dignity in the context of a relationship of responsibility, trust or power". ${ }^{23}$

Father: For the purpose of this paper, when referring to fathers, we mean biological fathers. Social fathers will be excluded.

Absent father: Will either be those alive and either known or unknown to the child.

Victimisation: Any subjectively reported or objectively measured experiences of gender-based violence within or outside relationships or interactions.

Perpetration: Will include men who have perpetrated GBV against women and girls and other men in the contexts of intimate relationships or non-intimate relationships, including those who perpetrate such violence against infants, children and lesbian, gay, bisexual and transgender populations.

Perpetrators: Will include anyone who has been reported to have perpetrated abuse on children whose fathers are absent.

Conflict settings: Are countries where armed conflict between opposing groupings is still ongoing. For the purpose of this study, a country is defined as a conflict setting even if the conflict was concentrated in a particular area at the time the study was conducted.

Post-conflict settings: Are settings where open fighting or warfare between opposing groupings has come to an end. For the purpose of this study, a country is defined as a conflict setting even if the conflict was concentrated in a particular area at the time the study was conducted.
Acknowledgements The authors would like to thank the Centre of Excellence for Human Development and the Faculty of Humanities at the University of the Witwatersrand for funding to carry out this study. Administrative research assistance to the project was gracefully undertaken by Oncemore Mbeve, to whom the authors extend their thanks. Furthermore, the authors would like to thank the College of Health Sciences, University of KwaZulu-Natal, for their support in setting up and conducting this research study.

Contributors YS, AM and MN conceptualised the study, while TPM-T and NK designed it. TPM-T and YS prepared the draft of the research proposal. All authors contributed to developing the background and planned output of the review, as well as the design of the study. TPM-T contributed to developing methods relating to review and synthesis of data-as well as the design of the sifting and data extraction processes. YS and TPM-T prepared the manuscript. All authors reviewed draft versions of the manuscript and approved the final version of the manuscript.

Funding Centre of Excellence for Human Development and the Faculty of Humanities at the University of the Witwatersrand.

Competing interests None declared.

Provenance and peer review Not commissioned; externally peer reviewed.

Data sharing statement The manuscript is a protocol for a scoping review. Thus, we do not have data currently. However, after the study has been carried out, we would be happy to share the data set.

Open Access This is an Open Access article distributed in accordance with the Creative Commons Attribution Non Commercial (CC BY-NC 4.0) license, which permits others to distribute, remix, adapt, build upon this work noncommercially, and license their derivative works on different terms, provided the original work is properly cited and the use is non-commercial. See: http:// creativecommons.org/licenses/by-nc/4.0/

\section{REFERENCES}

1. Fulu E, Warner X, Meidema S, et al. Why do some men use violence against women and how can we prevent it? Quantitative findings from the United Nations multi-country study on men and violence in Asia and the Pacific. Bangkok: UNDP, UNFPA, UN Women, UNV, 2013.

2. Garcia-Moreno C, Jansen HAFM, Ellsberg M, et al. WHO multi-country study on women's health and domestic violence against women: initial results on prevalence, health outcomes and women's responses. Geneva, Switzerland: World Health Organization, 2005

3. WHO. Understanding and addressing violence against women: intimate partner violence. Geneva: World Health Organization, 2012.

4. Maxwell L, Devries K, Zionts D, et al. Estimating the effect of intimate partner violence on women's use of contraception: a systematic review and meta-analysis. PLOS ONE 2014;10: e0118234.

5. Heise L, Ellsberg M, Gottemoeller M. Ending violence against women. Baltimore: Centre for Communications Programs, School of Public Health, Johns Hopkins University, 1999.

6. Ellsberg M, Heise L. Researching violence against women: a practical guide for researchers and activists. Washington DC, USA World Health Organization, 2005.

7. Jordan CE, Campbell R, Follingstad D. Violence and women's mental health: the impact of physical, sexual, and psychological aggression. Annu Rev Clin Psychol 2010;6:607-28.

8. Nduna M, Jewkes R. Undisclosed paternal identity in narratives of distress among young people in the Eastern Cape, South Africa. $J$ Child Fam Stud 2011;20:303-10.

9. East L, Jackson DJ, O'Brien L. Father absence and adolescent development: a review of literature. J Child Health Care 2006;10:283-95.

10. Holborn L. Fractured families: a crisis for South Africa. SAIIR fast facts. Johannesburg: South African Institute of Race Relations, 2011.

11. Padi T, Nduna M, Khunou G, et al. Defining absent, unknown and undisclosed fathers in South Africa. S Afr Rev Soc 2014;45:44-9.

12. Nduna $M$, Jewkes $R$. Denied and disputed paternity in teenage pregnancy: topical structural analysis of case studies of young women from the Eastern Cape Province. Soc Dynamics 2012;38:314-30.

13. Mavungu EM, Thompson-de Boor H, Mphaka K. "So we are ATM fathers": a study of absent fathers in Johannesburg. South Africa: 
Centre for Social Development in Africa, University of Johannesburg, 2013.

14. Mbatha K. Social support among black African women who have recently given birth: South African mothers' perspective. $N$ Voice Psychol 2012;8:3-16.

15. Townsend NW, Madhavan S, Garey A. Father presence in rural South Africa: incorporating social connection and life course experience. Meeting of the American Sociological Association Marriott Hotel, Loews Philadelphia Hotel, Philadelphia, 2005.

16. Nduna M, Sikweyiya Y. Silence from young women's narratives of absent, unknown and undisclosed fathers from Mpumalanga, South Africa. J Child Fam Stud 2015;24:536-45.

17. Nduna M. Factors that hinder the disclosure of the biological identity of a father to a child: South African mothers' perspective. J Feminist Fam Ther 2014;26:218-35.
18. Naidoo L, Sewpaul V. The life experiences of adolescent sexual offenders: factors that contribute to offending behaviours. Soc Work 2014;50:84-98.

19. Mathews S, Jewkes R, Abrahams N. 'I had a hard life': exploring childhood adversity in the shaping of masculinities among men who killed and intimate partner in South Africa. Br J Criminol 2011:51:960-77.

20. Wineburgh AL. Treatment of children with absent fathers. Child Adolesc Soc Work J 2000;17:255-73.

21. UN. Declaration on the eliminations of violence against women. Geneva, Switzerland: United Nations, 1993.

22. Ellsberg M, Heise L. Bearing witness: ethics in domestics violence research. Lancet 2002;359:1559-604.

23. World Health Organization. Report of the consultation on child abuse prevention, 29-31 March 1999. Geneva: WHO. 